\title{
Photobiomodulation Therapy for the Management of Patients With Inferior Alveolar Neurosensory Disturbance Associated With Oral Surgical Procedures: An Interventional Case Series Study
}

\author{
Neda Hakimiha ${ }^{\circledR}$, Amir Reza Rokn², Shima Younespour ${ }^{3}$, Neda Moslemi ${ }^{4 * \mathbb{C}}$ \\ ${ }^{1}$ Laser Research Center of Dentistry, Dentistry Research Institute, Tehran University of Medical Sciences, Tehran, Iran \\ ${ }^{2}$ Dental Implant Research Center, Dentistry Research Institute, Department of Periodontology, School of Dentistry, Tehran \\ University of Medical Sciences, Tehran, Iran \\ ${ }^{3}$ Assistant Professor, PhD of Biostatistics, Dentistry Research Institute, Tehran University of Medical Sciences, Tehran, Iran \\ ${ }^{4}$ Department of Periodontology, School of Dentistry, Tehran University of Medical Sciences, Tehran, Iran
}

\section{*Correspondence to Neda Moslemi, \\ DDS, MSc, School of Dentistry, Tehran University of Medical Sciences, North Kargar st, Tehran, Iran \\ Tel: +982188015088; Fax: +982188015800 Email: neda_moslemi@yahoo. com}

Published online December 30 2020

\begin{abstract}
Introduction: Inferior alveolar nerve (IAN) injury is a serious complication during intraoral surgeries We aimed to evaluate the outcome of photobiomodulation (PBM) therapy in patients with IAN injury associated with third molar or implant procedures.

Methods: Eight patients with an alteration of sensory function of the IAN after third molar or implant surgeries were enrolled in this case series study. The patients received 10 sessions of PBM therapy (810 nm diode laser, $200 \mathrm{~mW}, 10 \mathrm{~J} / \mathrm{cm}^{2}$ per point, three times a week). Pinprick (PP) and visual analogue scale (VAS) neurosensory tests were recorded at each treatment session and 14 days after the last treatment. The association between explanatory variables and the outcome of interest was analyzed using generalized estimating equations.

Results: The median percentage change of outcomes from the first to the last visit was as follows: VAS score: $+125.00 \%$ (range: 50.00 to 166.67 ); PP score: $+350 \%$ (range: 150 to 800 ). The duration of paresthesia was inversely correlated with changes in VAS and PP scores. No significant association was found between patients' gender or age and changes in VAS and PP scores.

Conclusion: Considering the limitations of this study, PBM with the parameters used in this study presented positive effects on neurosensory recovery in patients suffering from IAN injury associated with routine intraoral procedures. Patients with shorter duration of paresthesia tended to respond more favorably to PBM therapy.

Keywords: Low-level light therapy; Nerve regeneration; Inferior alveolar nerve; Photobiomodulation therapy.
\end{abstract}

\section{Introduction}

Peripheral nerve injuries during intraoral surgeries are challenging adverse events for both the patient and the surgeon. ${ }^{1}$ The inferior alveolar nerve (IAN) is the most commonly injured nerve during mandibular third molar surgery ${ }^{2}$ and dental implant procedure in the posterior mandible ${ }^{3}$ as the incidence of this complication has been estimated to occur up to $20 \%$ and $40 \%$ respectively. ${ }^{2,4}$ The primary risk factors include the difficulty of the procedure, initial surgeons' experience, and the close proximity of the mandibular third molar or implant fixture to the IAN. ${ }^{2}$

In cases of such nerve injuries, patients may experience different neurosensory alterations including hypoesthesia, hyperesthesia, paresthesia, and anesthesia. ${ }^{5}$ Moreover, these phenomena may result in reactive depression that can worsen the clinical status and lead to starting legal action against the doctor. ${ }^{3}$

At present, there is no defined protocol for the management of nerve injuries. In low to moderate nerve injuries named as neuropraxia and axonotmesis, medication and supportive physical therapy have been proposed for the reduction of symptoms and recovery time. ${ }^{6}$ Microneurosurgery is indicated in completely transected nerve injuries, i.e. neurotmesis. ${ }^{4}$ However, in most instances, complete rehabilitation is not achieved by any of these approaches. Recently, laser therapy or

Please cite this article as follows: Hakimiha N, Rokn AR, Younespour S, Moslemi N. Photobiomodulation therapy for the management of patients with inferior alveolar neurosensory disturbance associated with oral Surgical procedures: an interventional case series sudy. J Lasers Med Sci. 2020;11(suppl 1):S113-S118. doi:10.34172/jlms.2020.S18. 
photobiomodulation (PBM) has become an increasingly used treatment modality, especially in the areas of physical medicine and rehabilitation. PBM might offer beneficial results in cases with low to moderate nerve injuries. In addition, it has been proposed as an adjunctive method in severe nerve injuries. ${ }^{7}$

PBM is a non-thermallight therapy aiming at modulating tissue metabolism. These features are mediated by light absorption in cytochrome c oxidase (CCO) as the primary chromophore in mitochondria. The stimulated $\mathrm{CCO}$ induces an increase in electron transport chain activity and ATP synthesis. ${ }^{8}$ This process can activate several signaling pathways leading to the modulation of cell reactions. ${ }^{9}$ PBM has shown several positive effects on the regeneration of peripheral nerve injuries. These include axonal growth and myelinization, ${ }^{10}$ decrease or prevention of scar formation, ${ }^{11}$ decline of mononuclear inflammatory infiltration, ${ }^{12}$ upregulation of neurotrophic growth factors, ${ }^{13}$ improvement of functionality, ${ }^{14}$ and improved neurosensory recovery. ${ }^{15}$ Despite the promising finding in animal studies, there are conflicting results in human clinical nerve injury studies. ${ }^{16,17}$ This study aimed to evaluate the efficacy of PBM on neurosensory recovery in patients suffering from IAN disturbance associated with routine intraoral procedures.

\section{Materials and Methods \\ Patients}

The patients in the present study were selected from those referred to the clinic of the laser research center of dentistry, Tehran University of Medical Sciences between February 2018 and November 2019. Patients complaining of paresthesia of the lip and/or chin following mandibular third molar extraction or implant placement were enrolled. The patients were excluded if they had any relevant systemic disease, neurological disorders, infection at the site of injury, or any history of other interventions for the management of nerve injury. In addition, patients taking medications affecting the wound healing process were excluded. All patients provided signed informed consent to participate in this study.

\section{Assessment of IAN Paresthesia}

To determine the degree of the neurosensory deficit, visual analogue scale (VAS) and Pinprick (PP) tests were used as subjective and objective assessments respectively. These tests were examined at baseline and each treatment session, just before irradiation, and 14 days after the end of the treatment sessions.

\section{Pinprick Test}

The PP test included ten touches with an explorer tip (No. 23, shepherds hook single ended, Hu-Friedy Mfg. Co., Chicago, USA) on the extraoral soft tissues (lip and chin), while the patient's eyes were closed. The explorer was applied with moderate pressure to the lip ( 2 points) and the chin (eight points) for approximately 2-3 seconds with interval time of 30 seconds. The distance between the 2 points was considered about $10 \mathrm{~mm}$. The number of points in which the patient noticed the explorer tip touch was recorded in each session. The range of response was considered between $0-10 .{ }^{18}$

\section{Visual Analogue Scale Test}

The test was performed using a $10-\mathrm{cm}, 10^{\circ}$ line with divisions at $1-\mathrm{cm}$ intervals (The number 0 meant the complete absence of sensation and 10 at the extreme end indicated fully normal sensation). The patients were asked to choose a number between $0-10$ on the line at each testing. ${ }^{19}$

\section{Photobiomodulation Treatment}

All patients received 10 sessions of laser therapy every 48 hours (three times a week) with an 810-nm diodelaser (Fox, A.R.C laser, Nuremberg, Germany). The PBM protocol is presented in Table 1. In each session, the output power of the laser device was checked by a calibrated power meter (Laser Point s.r.1, Milano, Italy). A single practitioner experienced in the PBM field performed all irradiations (N. H.). Safety goggles were used by the practitioner and the patient during laser irradiation. The treatment was performed on several points including intra- and extraoral sites as follows: extraorally: two points on the lower lip, nine points on the chin, and one point on the region of mental foramen; intraorally: mandibular foramen, mental foramen region, one point on the buccal site of injury (perpendicular to the mandible body in the vestibular depth at the site of third molar or implant placement), and two points $10 \mathrm{~mm}$ anterior and posterior to the injured site at the same vertical position.

\section{Statistical Methods}

Data analyses were performed using statistical software SPSS 16 (SPSS Inc. Chicago, IL, USA) and JMP, Version

\begin{tabular}{ll} 
Table 1. PBM Protocol Used in This Study \\
\hline Parameter (Unit) & Laser Source \\
\hline wavelength & $810 \mathrm{~nm}$ \\
Manufacture & $\begin{array}{l}\text { Fox; A.R.C Laser, GmbH, } \\
\text { Nuremberg, Germany }\end{array}$ \\
Mode of irradiation & Continuous \\
Power (mW) & 200 \\
Beam spot size at target $\left(\mathrm{cm}^{2}\right)$ & 0.5 \\
Irradiance at target (mW/cm $\left.{ }^{2}\right)$ & 400 \\
Exposure time (s) & 25 per point \\
Radiant exposure $\left(\mathrm{J} / \mathrm{cm}^{2}\right)$ & 10 \\
Radiant energy $(\mathrm{J})$ & 5 \\
Application technique & Contact with mild pressure \\
Number of points irradiated & 17 points (12 extraoral, 5 intraoral) \\
Number and frequency of treatment & 10 sessions (3 times a week) \\
\hline
\end{tabular}


7 (SAS Institute Inc., Cary, NC, 1989-2007). Continuous variables were summarized as median, interquartile range (IQR: 25th- to 75th-percentile) and total range. Categorical data were reported as No. (\%). $P$ values less than 0.05 were considered statistically significant. The association between explanatory variables and the outcome of interest was analyzed using generalized estimating equation (GEE) in order to take into account the correlated nature of outcomes over time.

\section{Results}

Eight patients with paresthesia (4 women and 4 men) were recruited in this study. The median age of patients was 47.5 years (IQR: 44.25 to 56 ; range: 26 to 60 years). Six of these patients had paresthesia due to mandibular third molar surgery and 2 patients had paresthesia following implant placement (Table 2). The median duration of paresthesia was 2.25 months (IQR: 0.68 to 19 ; range: 0.23 to 26 months).

The median VAS and PP scores of patients with paresthesia in each time point are presented in Figure 1. Complete neurosensory recovery was observed in two patients with 7-day and 12-day duration of paresthesia according to both scores of VAS and PP on a 35-day follow-up visit. In addition, one of the patients had complete neurosensory recovery on a 35-day follow-up visit according to the VAS score with the 45-day duration of paresthesia.

On day 35 of the study, the median percentage change of VAS scores from the baseline was $+125.00 \%$ (range: $50.00 \%$ to $166.67 \%$ ). According to the GEE model, a significant increase was found in VAS scores over time of the study $(\beta=0.43, \mathrm{SE}=0.04,95 \% \mathrm{CI}$ for $\beta$ : 0.35 to 0.52 and $P<0.001)$. The duration of paresthesia was inversely correlated with VAS scores $(\beta=-0.08, S E=0.02,95 \%$ CI for $\beta$ : -0.11 to -0.04 and $P<0.0001)$. On day 35 of the study, the median percentage change of PP scores from the baseline was $+350 \%$ (range: $150 \%$ to $800 \%$ ). There was a significant increase in PP scores over time ( $\beta=0.62$, $\mathrm{SE}=0.06,95 \% \mathrm{CI}$ for $\beta: 0.51$ to 0.72 and $P<0.001$ ). Shorter
Table 2. Information Regarding the Patients Enrolled in the Study

\begin{tabular}{|c|c|c|c|c|c|}
\hline $\begin{array}{l}\text { Patient } \\
\text { No. }\end{array}$ & Sex & $\begin{array}{l}\text { Age } \\
(y)\end{array}$ & $\begin{array}{l}\text { Duration of } \\
\text { Paresthesia }\end{array}$ & Procedure & Referred Center \\
\hline 1 & M & 56 & $45 d$ & Implant & Private dental office \\
\hline 2 & M & 60 & $45 d$ & Implant & Private dental office \\
\hline 3 & $\mathrm{~F}$ & 26 & $7 d$ & $\begin{array}{l}\text { Third molar } \\
\text { surgery }\end{array}$ & Dental faculty \\
\hline 4 & M & 44 & 4 mon & Implant & Private dental office \\
\hline 5 & M & 45 & 3 mon & Implant & Private dental office \\
\hline 6 & $\mathrm{~F}$ & 50 & $12 \mathrm{~d}$ & Implant & Private dental office \\
\hline 7 & $\mathrm{~F}$ & 56 & 24 mon & $\begin{array}{l}\text { Third molar } \\
\text { surgery }\end{array}$ & Private dental office \\
\hline 8 & $\mathrm{~F}$ & 45 & 26 mon & Implant & Private dental office \\
\hline
\end{tabular}

M: male, F: female

duration of paresthesia was associated with a higher PP score $(\beta=-0.09, \mathrm{SE}=0.01,95 \% \mathrm{CI}$ for $\beta$ : -0.11 to -0.08 and $P<0.001)$. There was no significant association between individuals' age and both changes of VAS scores $(\beta=-0.04$, $\mathrm{SE}=0.02,95 \% \mathrm{CI}$ for $\beta$ : -0.08 to 0.002 and $P<0.06)$ and PP scores $(\beta=-0.04, \mathrm{SE}=0.02,95 \% \mathrm{CI}$ for $\beta$ : -0.07 to 0.002 and $P<0.07)$ over time. No significant association was found between patients' gender and both changes of VAS scores ( $\beta=0.20$ (male vs. female), $S E=0.63,95 \% \mathrm{CI}$ for $\beta$ : -1.04 to 1.45 and $P<0.75)$ and $P P$ scores $(\beta=0.41, S E=0.73,95 \% \mathrm{CI}$ for $\beta$ : -1.02 to 1.84 and $P<0.58$ ).

\section{Discussion}

There is a large variety of PBM protocols such as wavelength, energy density, power density, and treatment frequency used for healing purposes. ${ }^{19-21}$ Thus, up to now, it is not possible to obtain an informative and comprehensive instruction for the use of PBM in neurorehabilitation. ${ }^{22}$ The current study aimed to assess the efficacy of PBM in the sensory recovery of IAN in patients suffering from paresthesia associated with intraoral procedures. The results revealed that in all patients, neurosensory status improved after receiving PBM therapy. Moreover, the patients with shorter duration of paresthesia tended to

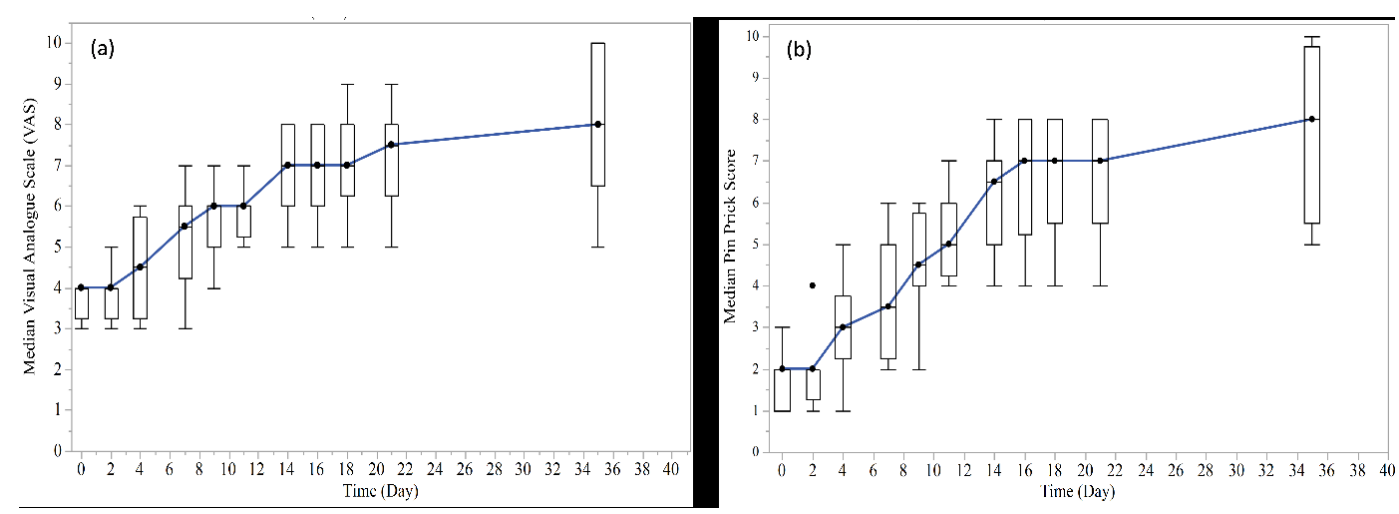

Figure 1. (a) Median VAS Scores and (b) Median Pinprick Scores of Patients (with Box plot) in Each Study Time Point. Note: VAS scores range from 0 to 10, considering a line with 0 indicating the complete absence of sensation and 10 at the extreme end expressing fully normal sensation; Pinprick score was recorded as the number of points in which the patient noticed the explorer tip touch in each session 
respond more favorably to $\mathrm{PBM}$ therapy. This finding is inconsistent with those of Pol et $\mathrm{al}^{21}$ and de Oliveira et $\mathrm{al}^{23}$ studies which showed a higher recovery rate of IAN regarding the VAS test in patients who received PBM within 6 months after IAN damage compared to those receiving it later. On the other hand, another case series reported successful results after PBM therapy in patients with history of more than one year clinical symptoms of lip/chin paresthesia. ${ }^{20}$ The number of PBM treatment sessions was set at 20 sessions that may be associated with achieving good results even in long term paresthesia.

It is important to note that the nerve recovery process may happen spontaneously between several weeks and one year post injuries depending on the severity of injuries. ${ }^{24}$ Thus, the results should be interpreted with caution in uncontrolled studies on patients with short duration of paresthesia.

Noteworthily, Santos et al. investigated the efficacy of PBM on IAN recovery in patients suffering from neurosensory disturbance associated with bilateral sagittal split osteotomy in a randomized split-mouth design study. Patients were divided into 2 groups: group 1 who received $\mathrm{PBM}$ during the short postoperative period (30 days) and group 2 treated during the late postoperative period (6 months to 1 year). The results showed sensory improvement in both experimental and control sides. However, the experimental side in both groups exhibited a marked improvement in sensory recovery during the follow-up period, and group 1 had the best results. ${ }^{25}$ The early beginning of PBM after nerve injury probably accelerates the neurosensory recovery by reducing the post-trauma inflammation and enhancing the regenerative process. ${ }^{13}$

In this study, we applied both objective and subjective tests for the assessment of neurosensory status. Most of the previous case series studies investigated the sensory recovery by mechanoceptive objective tests which assess the large myelinated fibers (A- $\alpha$ and A- $\beta$ axons), ${ }^{20,26}$ while the PP test is a nociceptive objective test which evaluates the integrity of cells innervated by small myelinated A- $\delta$ and $C$ fibers. ${ }^{27}$ The purpose of the inclusion of the subjective test (VAS) was to detect any kind of paresthesia not identified by objective tests. ${ }^{28}$

In this study, an 810-nm laser was used as the light source for PBM therapy. The $808-830 \mathrm{~nm}$ lasers are the most used wavelengths for the purpose of neurorehabilitation. ${ }^{29}$ This wavelength has a good penetration depth suitable for targets with a deep location like the IAN. ${ }^{30}$ Moreover, the $\mathrm{CCO}$ as the main chromophore in light absorption has the maximum absorption bands in the range of the near infra-red spectrum $(\sim 800 \mathrm{~nm}))^{31}$ To ensure the delivery of a sufficient dose at the level of the target, we should select proper dosimetry during PBM therapy. In this study, we applied $5 \mathrm{~J}$ energy and $10 \mathrm{~J} / \mathrm{cm}^{2}$ energy density per point. The effectiveness of PBM treatment using the energy density in the range of $6-10 \mathrm{~J} / \mathrm{cm}^{2}$ has been shown in the previous studies. ${ }^{19,20}$ It seems that the insufficient dose of the laser in PBM therapy was the reason for failure in the enhancement of neurosensory recovery in the study by Miloro et al. They applied total energy of $6 \mathrm{~J}$ and $3 \mathrm{~J}$ in 5 intra-oral and 8 extra-oral points respectively, ${ }^{16}$ which was much lower than that of studies with positive results. ${ }^{19,21}$

In the current study, we did not find any significant association between patients' age and the outcome scores. However, previous studies reported a higher sensory recovery rate in younger patients compared to older ones. ${ }^{15,32}$ This inverse relationship between age and regeneration capacity of peripheral nerves has been attributed to lower secretion of neurotrophic factors and reduction in terminal and collateral sprouting of regenerated fibers in older patients. ${ }^{33}$ In our study, the patients' median age was 47.5 years with only one patient under 30 years old. This age distribution could affect our results.

No difference in outcome scores was found related to gender in the present study. This finding is similar to that of de Oliveira et al study subsequent to the analysis of clinical records of patients receiving PBM therapy for the acceleration of IAN recovery after orthognathic or minor oral surgeries. ${ }^{34}$

\section{Conclusion}

Within the limitations of this study, PBM with the parameters used in the current study presented positive effects on IAN recovery in both subjective and objective neurosensory tests in patients complaining of neurosensory disturbance associated with routine intraoral procedures including third molar or implant procedures. Patients with shorter duration of paresthesia tended to respond more favorably to the PBM therapy.

\section{Ethical Considerations}

Informed consent for the execution of the procedure was obtained from all the patients.

\section{Conflict of Interests}

The authors have no conflict of interest, either directly or indirectly, in the laser devices or information listed in the article.

\section{Acknowledgments}

We gratefully thank the laser research center of dentistry, dentistry research institute, Tehran University of Medical Sciences for the cooperation in providing the diode laser.

\section{References}

1. Greenstein G, Carpentieri JR, Cavallaro J. Nerve damage related to implant dentistry: incidence, diagnosis, and management. Compend Contin Educ Dent. 2015;36(9):6529.

2. Auyong TG, Le A. Dentoalveolar nerve injury. Oral Maxillofac Surg Clin North Am. 2011;23(3):395-400. doi: 
10.1016/j.coms.2011.05.001.

3. Shavit I, Juodzbalys G. Inferior alveolar nerve injuries following implant placement - importance of early diagnosis and treatment: a systematic review. J Oral Maxillofac Res. 2014;5(4):e2. doi: 10.5037/jomr.2014.5402.

4. Rizzo S, Lupi SM, Zampetti P. Nerve damage resulting from oral surgery and medical legal implications. J Osseointegration. 2009;1(3):86-94. doi: 10.23805/ jo.2009.01.03.02.

5. Zuniga JR. Sensory outcomes after reconstruction of lingual and inferior alveolar nerve discontinuities using processed nerve allograft-A case series. J Oral Maxillofac Surg. 2015;73(4):734-44. doi: 10.1016/j.joms.2014.10.030.

6. Pogrel MA. Nerve damage in dentistry. Gen Dent. 2017;65(2):34-41.

7. Rochkind S. Laser photobiomodulation in neuroscience: From bench to bedside. Photomed Laser Surg. 2016;34(12):585-6. doi: 10.1089/pho.2016.4206.

8. de Freitas LF, Hamblin MR. Proposed mechanisms of photobiomodulation or low-level light therapy. IEEE J Sel Top Quantum Electron. 2016;22(3):7000417.

9. Hamblin MR. Mechanisms and mitochondrial redox signaling in photobiomodulation. Photochem Photobiol. 2018;94(2):199-212. doi: 10.1111/php.12864.

10. Mashhoudi Barez M, Tajziehchi M, Heidari MH, Bushehri A, Moayer F, Mansouri N, et al. Stimulation effect of low level laser therapy on sciatic nerve regeneration in rat. $J$ Lasers Med Sci. 2017;8(Suppl 1):S32-S37. doi: 10.15171/ jlms.2017.s7.

11. Rochkind S, Barrnea L, Razon N, Bartal A, Schwartz M. Stimulatory effect of He-Ne low dose laser on injured sciatic nerves of rats. Neurosurgery. 1987;20(6):843-7. doi: 10.1227/00006123-198706000-00004.

12. Cidral-Filho FJ, Martins DF, More AO, Mazzardo-Martins L, Silva MD, Cargnin-Ferreira E, et al. Light-emitting diode therapy induces analgesia and decreases spinal cord and sciatic nerve tumour necrosis factor-a levels after sciatic nerve crush in mice. Eur J Pain . 2013;17(8):1193-204. doi: 10.1002/j.1532-2149.2012.00280.x.

13. Hakimiha N, Dehghan MM, Manaheji H, Zaringhalam J, Farzad-Mohajeri S, Fekrazad R, et al. Recovery of inferior alveolar nerve by photobiomodulation therapy using two laser wavelengths: A behavioral and immunological study in rat. J Photochem Photobiol B. 2020;204:111785. doi: 10.1016/j.jphotobiol.2020.111785.

14. Andreo L, Soldera CA, Ribeiro BG, de Matos PRV, Sousa PB, de Alcantara Araujo Amorim WW, et al. Effects of photobiomodulation on functionality in wistar rats with sciatic nerve injury. Photochem Photobiol. 2019;95(3):87985. doi: $10.1111 /$ php. 13048 .

15. de Oliveira Martins D, Martinez dos Santos F, Evany de Oliveira M, de Britto LRG, Benedito Dias Lemos J, Chacur M. Laser therapy and pain-related behavior after injury of the inferior alveolar nerve: possible involvement of neurotrophins. J Neurotrauma. 2013;30(6):480-6. doi: 10.1089/neu.2012.2603.

16. Miloro M, Criddle TR. Does low-level laser therapy affect recovery of lingual and inferior alveolar nerve injuries? J Oral Maxillofac Surg. 2018;76(12):2669-75. doi: 10.1016/j. joms.2018.06.001.

17. Buchaim DV, Andreo JC, Ferreira Junior RS, Barraviera
B, Rodrigues AC, Macedo MC, et al. Efficacy of laser photobiomodulation on morphological and functional repair of the facial nerve. Photomed Laser Surg. 2017;35(8):442-9. doi: 10.1089/pho.2016.4204.

18. Sarikov R, Juodzbalys G. Inferior alveolar nerve injury after mandibular third molar extraction: a literature review. J Oral Maxillofac Res. 2014;5(4):e1. doi: 10.5037/ jomr.2014.5401.

19. Qi W, Wang Y, Huang YY, Jiang Y, Yuan L, Lyu P, et al. Photobiomodulation therapy for management of inferior alveolar nerve injury post-extraction of impacted lower third molars. Lasers in Dental Science. 2019;3:1-8.

20. Ozen T, Orhan K, Gorur I, Ozturk A. Efficacy of low level laser therapy on neurosensory recovery after injury to the inferior alveolar nerve. Head Face Med. 2006;2:3. doi: 10.1186/1746-160X-2-3.

21. Pol R, Gallesio G, Riso M, Ruggiero T, Scarano A, Mortellaro C, et al. Effects of superpulsed, low-level laser therapy on neurosensory recovery of the inferior alveolar nerve. J Craniofac Surg. 2016;27(5):1215-9. doi: 10.1097/ SCS.0000000000002757.

22. Zein R, Selting W, Hamblin MR. Review of light parameters and photobiomodulation efficacy: dive into complexity. J Biomed Opt. 2018;23(12):1-17. doi: 10.1117/1. JBO.23.12.120901.

23. de Oliveira RF, de Andrade Salgado DM, Trevelin LT, Lopes RM, da Cunha SR, Aranha AC, et al. Benefits of laser phototherapy on nerve repair. Lasers Med Sci. 2015;30(4):1395-406. doi: 10.1007/s10103-014-1531-6.

24. Cheung LK, Leung YY, Chow LK, Wong MC, Chan EK, Fok YH. Incidence of neurosensory deficits and recovery after lower third molar surgery: a prospective clinical study of 4338 cases. Int J Oral Maxillofac Surg. 2010;39(4):320-6. doi: 10.1016/j.ijom.2009.11.010.

25. Santos FT, Sciescia R, Santos PL, Weckwerth V, Dela Coleta Pizzol KE, Queiroz TP. Is low-level laser therapy effective on sensorineural recovery after bilateral sagittal split osteotomy? Randomized trial. J Oral Maxillofac Surg. 2019;77(1):164-73. doi: 10.1016/j.joms.2018.06.002.

26. Righesso LAR, Gil LF, Pantoja DS, Marin C, Granato R, Gil JN. Evaluation of neurosensory disturbances of the inferior alveolar nerve after intraoral verticosagittal ramus osteotomy. I Stomatol Oral Maxillofac Surg. 2018;119(3):192-5. doi: 10.1016/j.jormas.2018.02.005.

27. Antony PG, Sebastian A, Varghese KG, Sobhana CR, Mohan S, Soumithran CS, et al. Neurosensory evaluation of inferior alveolar nerve after bilateral sagittal split ramus osteotomy of mandible. J Oral Biol Craniofac Res. 2017;7(2):81-8. doi: 10.1016/j.jobcr.2017.03.004.

28. Shintani Y, Nakanishi T, Ueda M, Mizobata N, Tojyo I, Fujita S. Comparison of subjective and objective assessments of neurosensory function after lingual nerve repair. Med Princ Pract. 2019;28(3):231-5. doi: 10.1159/000497610.

29. Rosso MPO, Buchaim DV, Kawano N, Furlanette G, Pomini KT, Buchaim RL. Photobiomodulation therapy (PBMT) in peripheral nerve regeneration: A systematic review. Bioengineering (Basel). 2018;5(2):44. doi: 10.3390/ bioengineering 5020044 .

30. Hudson DE, Hudson DO, Wininger JM, Richardson BD. Penetration of laser light at 808 and $980 \mathrm{~nm}$ in bovine tissue samples. Photomed Laser Surg. 2013;31(4):163-8. doi: 
10.1089/pho.2012.3284.

31. Tsai SR, Hamblin MR. Biological effects and medical applications of infrared radiation.J Photochem Photobiol B. 2017;170:197-207. doi: 10.1016/j.jphotobiol.2017.04.014.

32. Midamba ED, Haanaes HR. Effect of low level laser therapy (LLLT) on inferior alveolar, mental and lingual nerves after traumatic injury in 15 patients. A pilot study. Laser Ther. 1993;5(2):89-94. doi: 10.5978/islsm.93-OR-10.
33. Verdu E, Ceballos D, Vilches JJ, Navarro X. Influence of aging on peripheral nerve function and regeneration. $J$ Peripher Nerv Syst. 2000;5(4):191-208. doi: 10.1046/j.15298027.2000.00026.x.

34. de Oliveira RF, da Silva AC, Simoes A, Youssef MN, de Freitas PM. Laser therapy in the treatment of paresthesia: A retrospective study of 125 clinical cases. Photomed Laser Surg. 2015;33(8):415-23. doi: 10.1089/pho.2015.3888. 